(1)

CrossMark

\title{
Incidental venous thromboembolism, detected by chance, but still venous thromboembolism
}

\author{
Guy Meyer and Benjamin Planquette
}

Affiliation: Division of Respiratory Disease, APHP Centre, Université Paris Descartes, Université de Paris, Paris, France.

Correspondence: Guy Meyer, Service de Pneumologie, Hôpital Européen Georges Pompidou, 20 rue Leblanc, 75015 Paris, France. E-mail: guy.meyerdaphp.fr

@ERSpublications

Incidental venous thromboembolism and clinically suspected venous thromboembolism should receive the same anticoagulant treatment http://bit.ly/2NQ418U

Cite this article as: Meyer G, Planquette B. Incidental venous thromboembolism, detected by chance, but still venous thromboembolism. Eur Respir J 2020; 55: 2000028 [https://doi.org/10.1183/13993003.000282020].

Incidental venous thromboembolism (VTE) refers to deep venous thrombosis or pulmonary embolism (PE) diagnosed on an imaging test, usually computed tomography, performed for another reason than the clinical suspicion of VTE. Incidental VTE is particularly frequent in patients with cancer who have an increased risk of VTE and who underwent frequent imaging tests for staging purposes and assessment of anticancer treatment.

Incidental VTE is usually opposed to symptomatic VTE but these terms may be misleading by suggesting that incidental VTE is mostly asymptomatic. In fact, most patients with incidental VTE have VTE-related symptoms. A more comprehensive wording may use the terms "clinically suspected" instead of symptomatic and "clinically unsuspected" instead of incidental.

Clinicians and researchers are faced with three main questions regarding clinically unsuspected VTE; 1) is the diagnosis reliable? 2) Are clinically unsuspected VTE events less severe than clinically suspected VTE events? And 3) is it worth treating unsuspected VTE as suspected VTE?

\section{Is the diagnosis of clinically unsuspected VTE reliable?}

Clinically unsuspected VTE is, by definition, diagnosed on an imaging test not aimed at diagnosing VTE. For PE, the quality of the injection matters and a suboptimal injection of the pulmonary arteries has been associated with an increased risk of indeterminate result [1]. Two studies, however, do not support this hypothesis. In one study, the kappa value between two expert readers and a non-expert interpretation was 0.93 , suggesting a good reproducibility and all 62 clinically unsuspected PEs diagnosed by a non-expert radiologist were confirmed by two experts, suggesting that the diagnosis of clinically unsuspected PE is reliable [2]. Of note, only one patient in this study had an isolated sub-segmental PE [2]. In another study, the agreement with regard to the most proximal location of clinically unsuspected PE was very good but decreased to moderate for subsegmental clots. In this study, the diagnosis of subsegmental PE was not confirmed by expert reading in only one (3\%) among 29 patients originally diagnosed with a sub-segmental PE, suggesting again that the diagnosis of clinically unsuspected PE is reliable even at the subsegmental level [3]. 


\section{Are clinically unsuspected VTE events less severe than clinically suspected VTE events?}

Clinically unsuspected PE is associated with more distal clots than clinically suspected PE [3, 4], and patients with clinically unsuspected PE appear to have a better prognosis than patients with clinically suspected PE, but this is critically dependent on the presence of symptoms. In patients with clinically unsuspected PE, the presence of symptoms adversely affects survival [5]. In the EPIPHANY study, patients with cancer and PE were divided into three groups, those with clinically suspected PE, those with clinically unsuspected PE and no symptoms attributable to PE, and a third group with clinically unsuspected PE who retrospectively had symptoms related to PE. Patients with clinically suspected PE were more likely to have a syncope or a heart rate $>100$ beats per min than symptomatic patients with clinically unsuspected $\mathrm{PE}$, but 90-day mortality rates were comparable in these two groups and higher than in the group of asymptomatic patients [6].

\section{Is it worth treating unsuspected VTE as suspected VTE?}

Current guidelines suggest the same therapeutic approach in patients with clinically unsuspected VTE and in patients with clinically suspected VTE $[7,8]$. These guidelines are mainly based on the results of retrospective cohort studies, suggesting that the risk of recurrent VTE during anticoagulant treatment is comparable in these two groups of patients. In cancer patients, even clinically unsuspected isolated subsegmental PE is associated with a substantial risk of recurrence during anticoagulant treatment [9]. The significant risk of recurrent VTE in patients with cancer and clinically unsuspected VTE has been confirmed in a recent multicentre prospective cohort study [10] and by the results of the study reported by Mulder et al. [11] in this issue of the European Respiratory Journal. In this latter study, the risk of recurrent VTE was numerically lower in patients with incidental VTE but the difference with patients with so-called symptomatic VTE was not statistically significant. These results were obtained in the context of a prospective multicentre therapeutic trial with centralised blinded adjudication of outcomes [11].

On aggregate, the available evidence strongly supports that incidental VTE and clinically suspected VTE share common risk factors and deserve the same therapeutic approach. The substantial rate of clinically unsuspected VTE in cancer patients also calls for a reduced index of suspicion by oncologists, allowing diagnosing VTE and especially PE before signs of haemodynamic instability occur.

Conflict of interest: G. Meyer reports grants and non-financial (travel) support from Leo Pharma, BMS-Pfizer, Stago and Bayer Healthcare, outside the submitted work. B. Planquette has nothing to disclose.

\section{References}

1 Jones SE, Wittram C. The indeterminate CT pulmonary angiogram: imaging characteristics and patient clinical outcome. Radiology 2005; 237: 329-337.

2 den Exter PL, van der Hulle T, Hartmann IJ, et al. Reliability of diagnosing incidental pulmonary embolism in cancer patients. Thromb Res 2015; 136: 531-534.

3 Bleker SM, Beenen LF, Di Nisio M, et al. Incidental pulmonary embolism in cancer patients: interobserver agreement on the diagnosis and extent with a focus on distal clots. Thromb Res 2016; 147: 46-51.

4 Sahut D'Izarn M, Caumont Prim A, Planquette B, et al. Risk factors and clinical outcome of unsuspected pulmonary embolism in cancer patients: a case-control study. J Thromb Haemost 2012; 10: 2032-2038.

5 O'Connell CL, Razavi PA, Liebman HA. Symptoms adversely impact survival among patients with cancer and unsuspected pulmonary embolism. J Clin Oncol 2011; 29: 4208-4209.

6 Font C, Carmona-Bayonas A, Beato C, et al. Clinical features and short-term outcomes of cancer patients with suspected and unsuspected pulmonary embolism: the EPIPHANY study. Eur Respir J 2017; 49: 1600282.

7 Kearon C, Akl EA, Comerota AJ, et al. Antithrombotic therapy for VTE disease: Antithrombotic Therapy and Prevention of Thrombosis, 9th ed: American College of Chest Physicians Evidence-Based Clinical Practice Guidelines. Chest 2012; 141: e419S-e494S.

8 Konstantinides SV, Meyer G, Becattini C, et al. 2019 ESC Guidelines for the diagnosis and management of acute pulmonary embolism developed in collaboration with the European Respiratory Society (ERS): The Task Force for the diagnosis and management of acute pulmonary embolism of the European Society of Cardiology (ESC). Eur Respir J 2019; 54: 1901647.

9 van der Hulle T, den Exter PL, Planquette B, et al. Risk of recurrent venous thromboembolism and major hemorrhage in cancer-associated incidental pulmonary embolism among treated and untreated patients: a pooled analysis of 926 patients. J Thromb Haemost 2016; 14: 105-113.

10 Kraaijpoel N, Bleker SM, Meyer G, et al. Treatment and long-term clinical outcomes of incidental pulmonary embolism in patients with cancer: an international prospective cohort study. J Clin Oncol 2019; 37: 1713-1720.

11 Mulder FI, Di Nisio M, Ay C, et al. Clinical implications of incidental venous thromboembolism in cancer patients. Eur Respir J 2020; 55: 1901697. 